\title{
Community Screening for Diseases - A Useful Public Health Tool
}

\author{
Tahir Masood Ahmad
}

Pakistan is the $5^{\text {th }}$ most populous country in the world and is $36^{\text {th }}$ largest nation by total area. Pakistan's overall health issues are protean and comprise of a dual burden of communicable and non-communicable diseases (NCD), large child population (35.4\% of population under 15$)^{1}$, high maternal mortality rate $(178 / 100,000$ live births) $)^{2}$ abysmal under-5 mortality rate (79/1000 live births $)^{3}$, widespread malnutrition in children $(44 \%$ stunting, $31 \%$ underweight) 4 , and highly inadequate provisions in terms of equitable healthcare. It is one of the two countries in the world where polio is still endemic. Pakistan's percentage of people living below poverty line continues to be around $24.3,5$ which directly and indirectly contributes to ill health.

In the conventional healthcare systems, there is a critical gap between the curative medicine and the community services. There is an emerging evidence that clinicalcommunity linkage improves health outcomes and reduces costs. In a resource-constrained country like Pakistan, where only 0.5 to $0.8 \%$ of GDP is spent on health ${ }^{6}$ (against the WHO benchmark of at least $6 \%$ of the GDP), it is essential that we lay emphasis on preventive health services at the community level. 'It is better to prevent than cure' is an old adage since the time of Hippocrates. The scarce resources need to be utilised judiciously and effectively. Early diagnosis of disease by community screening helps in instituting early treatment as well as reducing the morbidity and mortality.

Screening is a strategy used in a population to detect an as-yet undiagnosed disease in apparently asymptomatic individuals, so as to start early interventions for making an impact on reduction of death and disability. ${ }^{7}$ Conditions which can have substantial effect on the health of individual and which can be detected by a good screening test and treated effectively, are the ideal ones for which screening programmes should be in place. In an ideally functioning health system, these programmes should reach those who can benefit; and the health system is efficient enough to do appropriate interventions and monitor compliance. With community screening programmes, there is tremendous opportunity

The Children's Hospital and The Institute of Child Health, Lahore, Pakistan

Correspondence: Prof. Tahir Masood Ahmad, The Children's

Hospital and The Institute of Child Health, Lahore, Pakistan

E-mail: tahirmas@hotmail.com

Received: September 17, 2018; Accepted: December 05, 2018 to save years of life and improve the quality of life. These programmes also empower the community to take care of their own health and they also become more proactive in their health. Evidence-based preventive services can be instituted for chronic conditions such as diabetes mellitus, heart diseases, hypertension, obesity, visual disorders, mental health conditions, infectious diseases like hepatitis, HIV, and vaccine preventable diseases and certain cancers like cervical cancer, colorectal cancer, prostatic cancer and breast cancer. Notwithstanding the fact that screening can be fraught with certain adverse effects like overdiagnosis and misdiagnosis, its benefits far outweigh the disadvantages, if any.

There are several types of screening which are applicable in different situations. Initial screening programmes were devised using the Wilson-Jugner criteria ${ }^{8}$ developed by World Health Organization on the basis of two landmark reports about population screening. However with the introduction of genomic medicine and other new techniques, these criteria were modified later in 2008.9

A major component of community health screening is the newborn screening (NBS), which has been very widely used and has been recognised internationally as an essential preventive public health programme for early detection of certain disorders of newborn, which contribute to the morbidity and mortality of infants. Early diagnosis and treatment of various genetic, metabolic, endocrine, hematological or congenital disorders help in significant reduction of death and disability. 10 Infants are screened shortly after birth for a number of conditions which do not have overt symptoms in the immediate neonatal period but have the propensity to cause irreversible damage before they become clinically detectable. These may also lead to sudden death in the newborns and remain undiagnosed. These conditions, however, can be picked up early and treated effectively with the screening programmes. NBS was first introduced in the United States of America in 1960's by Dr. Robert Guthrie, initially for phenylketonuria (PKU). ${ }^{11}$ Guthrie was also pioneer in introducing the collection of blood on filter paper, making the transportation of samples very easy; and it became feasible to do the screening on a large scale. More and more conditions in the newborn period are now being screened, the number of which is variable in different countries of the world depending on prevalence of these conditions, available resources, and the legal implications. With available diagnostic techniques like tandem mass spectrometry, the number of diseases which can be included in the 
screening programme has gone over 50.12 Screening of newborns for hearing deficit or for congenital heart disease using appropriate methodology has also been proven beneficial. ${ }^{13}$ Genetic studies are now being used as a routine primary screening tool; and the screening has shifted closer to diagnosis.

Screening programmes however, require a comprehensive healthcare system and very vast and elaborate infrastructure with expertise in public health, laboratory services; and healthcare management. Community screening is an established programme in the developed world; but for the developing world, its establishment is a major challenge in the presence of major public health issues like infectious diseases, malnutrition, appalling maternal and child health, a heavy burden of NCD, and population control.

Pakistan faces the major health problems with a population of 207.74 million according to 2017 census with a growth rate of 2.10. More emphasis is on curative health. Apart from National EPI (Expanded Program on Immunization), acute flaccid paralysis (AFP) surveillance as part of global polio eradication initiative and patchy interventions like hepatitis, tuberculosis and malaria control programme, there hasn't been any other significant emphasis on preventive services. There is no newborn screening programme at national level. The most widely done NBS in Pakistan is for congenital hypothyroidism, which is being followed in the various centres across the country as isolated efforts. ${ }^{14}$ Pakistan has failed in achieving the targets of Millennium Development Goals (MDGs), but has vowed to uphold its commitment in achieving even stiff Sustainable Development Goals (SDGs)15, for which Pakistan will have to make a leap forward in many areas of which community health carries a significant importance. If we are to succeed in achieving the SDGs, the focus should be on prevention of diseases like tuberculosis, AIDS, malaria and other communicable and non communicable diseases (NCD). Promotion of good health and wellbeing is crucial for progress across multiple goals including alleviation of poverty and hunger. Pakistan can improve its health indicators by providing evidencebased, targeted and cost effective disease-screening and prevention programmes. We have very high consanguinity rate with consequently high incidence of hereditary diseases, especially autosomal recessive conditions. The treatment of these diseases is either not easily available or requires substantial health resources. When the world has taken up the task of sustainable development, Pakistan also needs to think about utilisation of its meager resources in the most cost effective manner. It is, therefore, imperative that NBS and community screening of selective diseases is started at the national level for which prevention is available and effective and less expensive treatment is started early, which will prove to be an effective public health intervention. This will also help public health people to make tough decisions about allocation of limited resources in a more logical and sustainable way, and the big challenge of providing the citizens of Pakistan with a basic package of essential and equitable health services can be met forthwith.

\section{REFERENCES}

1. United Nation's Statistics Division - Demographics and Social Statistics 2017 unstats.un.org/unsd/demographic/products/dyb

2. Pakistan Demographics Profile 2018 - IndexMundi https://www. indexmundi.com/pakistan/demographics_profile.html.

3. State of the World's Children 2017 statistical tables - UNICEF DATA https://data.unicef.org/resources/state-worlds-children2017-statistical-tables

4. Bhutta Z, Soofi S, Habib A, Hussain M. Pakistan National Nutrition Survey, 2011.

5. Asian Development Bank. (n.d.). Poverty in Pakistan. November 2016. https://www.adb.org/countries/pakistan/poverty

6. Pakistan Economic Survey 2016-17 Chapter 11, Health and Nutrition Page 187.

7. Woolf $\mathrm{SH}$. The power of prevention and what it requires. $J \mathrm{Am}$ Med Assoc 2008; 299:2437-9.

8. Wilson JMG, Jungner G. Principles and practice of screening for disease (1968). WHO Chronicle. Geneva: World Health Organization. 22 (11): 473 Public Health Papers, \#34.

9. Anne Andermann, Ingeborg Blancquaert, Sylvie Beauchamp, Véronique Déry revisiting Wilson and Jungner in the genomic age: A review of screening criteria over the past 40 years: Bulletin of the World Health Organization; 2008 Volume 86, Number 4, April 2008, 241-320 http://www.who.int/ bulletin/ volumes/86/4/07-050112/en/

10. Pollitt RJ. Newborn blood spot screening: New opportunities, old problems. J Inherit Metab Dis 2009; 32:395-9.

11. Gonzalez J, Willis MS. Clinical chemistry / microbiology. Lab Med 2009; 40:748-9.

12. Schoen EJ, Baker JC, Colby CJ. Cost-benefit analysis of universal tandem mass spectrometry for newborn screening. Pediatrics 2002; 110:781-6.

13. Thangaratinam S, Brown K, Zamora J, Khan KS, Ewer AK. Pulse oximetry screening for critical congenital heart defects in asymptomatic newborn babies: A systematic review and metaanalysis. Lancet 2012; 379: 2459-64.

14. Afroze B, Humayun NK, Qadir M. Newborn screening in Pakistan - Lessons from a hospital based congenital hypothyroidism screening programme: Ann Acad Med Singapore 2008; 37(suppl 3):114-6.

15. Sustainable Development Goals (SDGs) 2015-2030 June 2016 www.lead.org.pk/lead/attachments/SDGFlyer.pdf 